\title{
Corf $950112-3$
}

UCRL-JC-119843

PREPRINT

\section{Weakly Relativistic Modeling of Refraction and Absorption for Waves with Small $N_{\|}$}

\author{
Gary R. Smith, L.D. Pearlstein, A.H. Kritz, \\ I.P. Shkarofsky, A. Pochelon, A. Hirt, O. Sauter, T. Goodman, \\ G. Giruzzi, and J.L. Segui \\ This paper was prepared for publication in the \\ Proceedings of the Ninth Joint Workshop \\ on Electron Cyclotron Emission \& Electron Cyclotron Resonance Heating \\ Borrego Springs, California \\ January 22-26, 1995
}

January 1995

This is a preprint of a paper intended for publication in a journalorproceedings. Since changes may be made before publication, this preprint is made available with the understanding that it will not be cited or reproduced without the permission of the. author. 


\section{DISCLAIMER}

This document was prepared as an account of work sponsored by an agency of the United States Government. Neither the United States Government nor the University of California nor any of their employees, makes any warranty, express or implied, or assumes any legal liability or responsibility for the accuracy, completeness, or usefulness of any information, apparatus, product, or process disclosed, or represents that its use would not infringe privately owned rights. Reference herein to any specific commercial products, process, or service by trade name, trademark, manufacturer, or otherwise, does not necessarily constitute or imply its endorsement, recommendation, or favoring by the United States Government or the University of California. The views and opinions of authors expressed herein do not necessarily state or reflect those of the United States Government or the University of California, and shall not be used for advertising or product endorsement purposes. 


\section{DISCLAIMER}

Portions of this document may be illegible in electronic image products. Images are produced from the best available original document. 


\title{
Weakly Relativistic Modeling of Refraction and Absorption for Waves with Small $N_{\|}$
}

\author{
Gary R. Smith and L.D. Pearlstein \\ Lawrence Livermore National Laboratory, University of California \\ Livermore, California 94551, USA \\ A.H. Kritz \\ Lehigh University, Bethlehem, Pennsylvania 18015, USA \\ I.P. Shkarofsky \\ MPB Technologies, Inc., Pointe Claire, Québec H9R 1E9, Canada
}

A. Pochelon, A. Hirt, O. Sauter, and T. Goodman

CRPP-EPFL, Association EURATOM-Switzerland

Lausanne, Switzerland

G. Giruzzi and J.L. Ségui

Association EURATOM-CEA, Cadarache, France

Transmission measurements for waves near the fundamental and harmonics of the electron-cyclotron frequency indicate that propagation and absorption is not always correctly described when ray trajectories are obtained using cold-plasma analysis. Improved methods have been developed for evaluating the Shkarofsky functions, which appear in the weakly relativistic approximation of the dielectric tensor, for small parallel index of refraction. Computational results for vertical third-harmonic $\mathrm{X}$-mode propagation in Tore Supra show strong, warm-plasma refraction effects that qualitatively agree with experimental observations.

DISTRIBUTION OF THIS DUCUMENT IS UNLIMITED WN

DISTRIEUTION UF THIS UUCUMENT IS UNLIMITED

MASTER 


\section{Motivation}

This work is motivated by recent experiments, especially those in the Tore Supra tokamak, that suggest significant, warm-plasma refraction effects of waves near the fundamental and harmonics of the electron-cyclotron frequency. ${ }^{1-3}$ These works, as well as earlier ones, have shown that a relativistic treatment of the wave-particle resonance condition is necessary for accurate treatment of both refraction and absorption, even for plasmas with temperatures as low as $1 \mathrm{keV}$. Further motivation comes from plans for high-power electron-cyclotron heating (ECH) experiments in the TCV tokamak using extraordinary-mode ( $\mathrm{X}$-mode) waves at the second and third harmonics. ${ }^{4}$ TCV will employ ECH to create broad current profiles necessary for vertical stability of highly. elongated plasmas. Accurate prediction of wave propagation and absorption is therefore important to the success of TCV experiments. Warm-plasma refraction effects also have potential importance for the much hotter International Thermonuclear Experimental Reactor (ITER) for some ECH launch scenarios.

\section{Evaluation of Shkarofsky Functions $\mathcal{F}_{q}$}

This paper describes recent progress towards accurate treatment of refraction and absorption near cyclotron harmonics within the weakly relativistic approximation. ${ }^{5}$ In that approximation, the dielectric tensor is expressed in terms of a set of complexvalued functions $\mathcal{F}_{q}$ and their derivatives. These functions are defined by

$$
\begin{gathered}
\mathcal{F}_{q}(\phi, \psi) \equiv-i \exp \left(-\psi^{2}\right) \int_{0}^{\infty} d t(1-i t)^{-q} \exp \left[-i \phi^{2} t+\frac{\psi^{2}}{(1-i t)}\right] \\
q=\frac{1}{2}, \frac{3}{2}, \frac{5}{2}, \ldots, \frac{13}{2}
\end{gathered}
$$

The two parameters $\psi$ and $\phi$ are given by

$$
\begin{gathered}
\psi \equiv N_{\|} \sqrt{\mu / 2}, \quad \mu \equiv m c^{2} / T_{e} \\
\phi^{2} \equiv \psi^{2}-\mu \delta, \quad \delta \equiv 1-n \Omega / \omega
\end{gathered}
$$

where $\Omega$ is the cyclotron frequency, and $n$ is the cyclotron-harmonic number that appears in the dielectric tensor as a summation index. For real wave frequency $\omega$ and real parallel index of refraction $N_{\|}$, as assumed here, the parameters $\psi$ and $\phi^{2}$ are both real.

Depending on the values of $\phi$ and $\psi$, different evaluation methods and recursion relations are used for numerical calculation of $\mathcal{F}_{q}$, because each method is accurate 
in a region of the $\phi-\psi$ plane but inaccurate or inapplicable outside that region. The recursion relation

$$
\mathcal{F}_{q+2}=\left(1+\phi^{2} \mathcal{F}_{q}-q \mathcal{F}_{q+1}\right) / \psi^{2}, \quad q \geq \frac{1}{2}
$$

with starting values

$$
\begin{gathered}
\mathcal{F}_{\frac{1}{2}}=-Z^{+} / \phi, \quad \mathcal{F}_{\frac{3}{2}}=-Z^{-} / \psi \\
Z^{ \pm}=\frac{1}{2}[Z(\psi-\phi) \pm Z(-\psi-\phi)]
\end{gathered}
$$

was given by Krivenski and Orefice. ${ }^{6}$ Here, $Z$ denotes the standard plasma dispersion function. ${ }^{7}$ This method is useful for $\psi \gtrsim 1$. For small $N_{\|}$, and thus small $\psi$, this method suffers from loss of accuracy in the calculation of $\mathcal{F}_{q}$ for high $q$ starting from low $q$ (forward recursion). Bindslev ${ }^{8}$ overcame this problem by using the recursion relation iteratively to allow starting at high or intermediate $q$. Borie ${ }^{9}$ also overcame numerical problems at small $\psi$.

In the remainder of this section, we describe methods used in this work to evaluate the functions $\mathcal{F}_{q}$ for $\psi<3$. For brevity, we do not describe the methods, which are similar to those described below, that are used to evaluate the first through fourth derivatives of $\mathcal{F}_{q}$ with respect to $\phi^{2}$. Description of the accuracy and applicability of these methods, which is generally most challenging for the fourth derivative at the highest $q$ required, is also beyond the scope of this paper.

For $\phi^{2} \lesssim 10$ and $\psi<3$, we use the expression ${ }^{5}$

$$
\mathcal{F}_{q}(\phi, \psi)=\exp \left(-\psi^{2}\right) \sum_{\ell=0}^{\infty} F_{q+\ell}\left(-\phi^{2}\right) \psi^{2 \ell} / \ell !,
$$

in which the coefficients are Dnestrovsky functions $F_{q}$ and are found from

$$
F_{\frac{3}{2}}\left(-\phi^{2}\right)=-Z^{\prime}(-\phi) \text { and } F_{q+1}=\left(1+\phi^{2} F_{q}\right) / q, \text { for } q>\frac{3}{2} \text {. }
$$

The accuracy of this method degrades as $\phi^{2}$ becomes large, because the Dnestrovsky functions become inaccurate.

For $\phi^{2} \gtrsim 10$ and $\psi<3$, we use a method that is, to the best of our knowledge, published here for the first time. This new method is necessary, because the asymptotic series given in Eq. (A4) of Ref. 10 can only be used for very large values of $\phi^{2}$ $(\gtrsim 150)$. Thus for $10 \lesssim \phi^{2} \lesssim 150$, we evaluate the series

$$
\mathcal{F}_{q}(\phi, \psi)=\sum_{m=0}^{\infty} A_{m, q}(\phi) \psi^{2 m}
$$


in which the coefficients are found from derivatives of the $Z$ function using starting values

$$
A_{m, \frac{1}{2}}=-\frac{1}{\phi} \frac{Z^{(2 m)}(-\phi)}{(2 m) !}, \quad A_{m, \frac{3}{2}}=-\frac{Z^{(2 m+1)}(-\phi)}{(2 m+1) !}
$$

and the recursion relation

$$
A_{m, q}=\phi^{2} A_{m+1, q-2}-(q-2) A_{m+1, q-1}, \quad q=\frac{5}{2}, \frac{7}{2}, \ldots
$$

This method requires evaluation of very high order derivatives of $Z(-\phi)$ (at least 25 th order) for values of $-\phi$ along the real or positive imaginary axes. These high derivatives are calculated by methods similar to those described in App. A of Ref. 11.

The evaluation methods appropriate for small $\psi$, which are described in this section, have been installed recently in the TORCH ray-tracing code ${ }^{12}$ and used to obtain the results in this paper.

\section{Vertical X3 Propagation in Tore Supra}

In modeling vertical third-harmonic X-mode propagation in Tore Supra, we use a simple equilibrium model with unshifted, circular flux surfaces. The central magneticfield strength is $B=1.3 \mathrm{~T}$, the central density and temperature are $n_{e}(0)=3 \times$ $10^{19} \mathrm{~m}^{-3}$ and $T_{e}(0)=1 \mathrm{keV}$, and profiles of $n_{e}(r)$ and $T_{e}(r)$ are parabolic. Rays are launched from the location of the lower horn with angles with respect to the vertical ranging from $-6^{\circ}$ to $0^{\circ}$. The upper (receiving) horn is represented by an absorbing rectangle with the actual location and size $(4.4 \mathrm{~cm} \times 6.6 \mathrm{~cm})$ of the horn mouth.

Figure 1 shows a comparison of the results of ray tracing in the cold-plasma and weakly relativistic approximations. Warm-plasma effects have caused refraction away from the resonance on both the high- and low-field sides, which has opened a gap in the transmitted power pattern. On the high-field side there is absorption and focusing of power. Focusing on the low-field side is also present and leads to higher received power in the warm-plasma calculation than in the cold plasma.

The magnitude of the warm-plasma refraction effects can be seen clearly in Fig. 2. As a function of launch angle, we show the radial position $R$ that a ray has when it has reached the vertical position $Z$ of the receiving horn. The three curves show the results with no plasma (dashes), cold plasma (solid), and warm plasma (circles). Results for cold and warm plasma agree, as they must, away from resonance. Note that the ray launched at $\theta=-2.6^{\circ}$ grazes the resonance just to the low-field side. In a small interval near $R=247 \mathrm{~cm}$, a horizontal line at a given value of $R$ intersects the warm-plasma curve (circles) at two launch angles in the interval $-2.6^{\circ}<\theta<-1.8^{\circ}$. (A third launch angle near $\theta=-2.7^{\circ}$ also intersects $R=247 \mathrm{~cm}$, but the portion of 


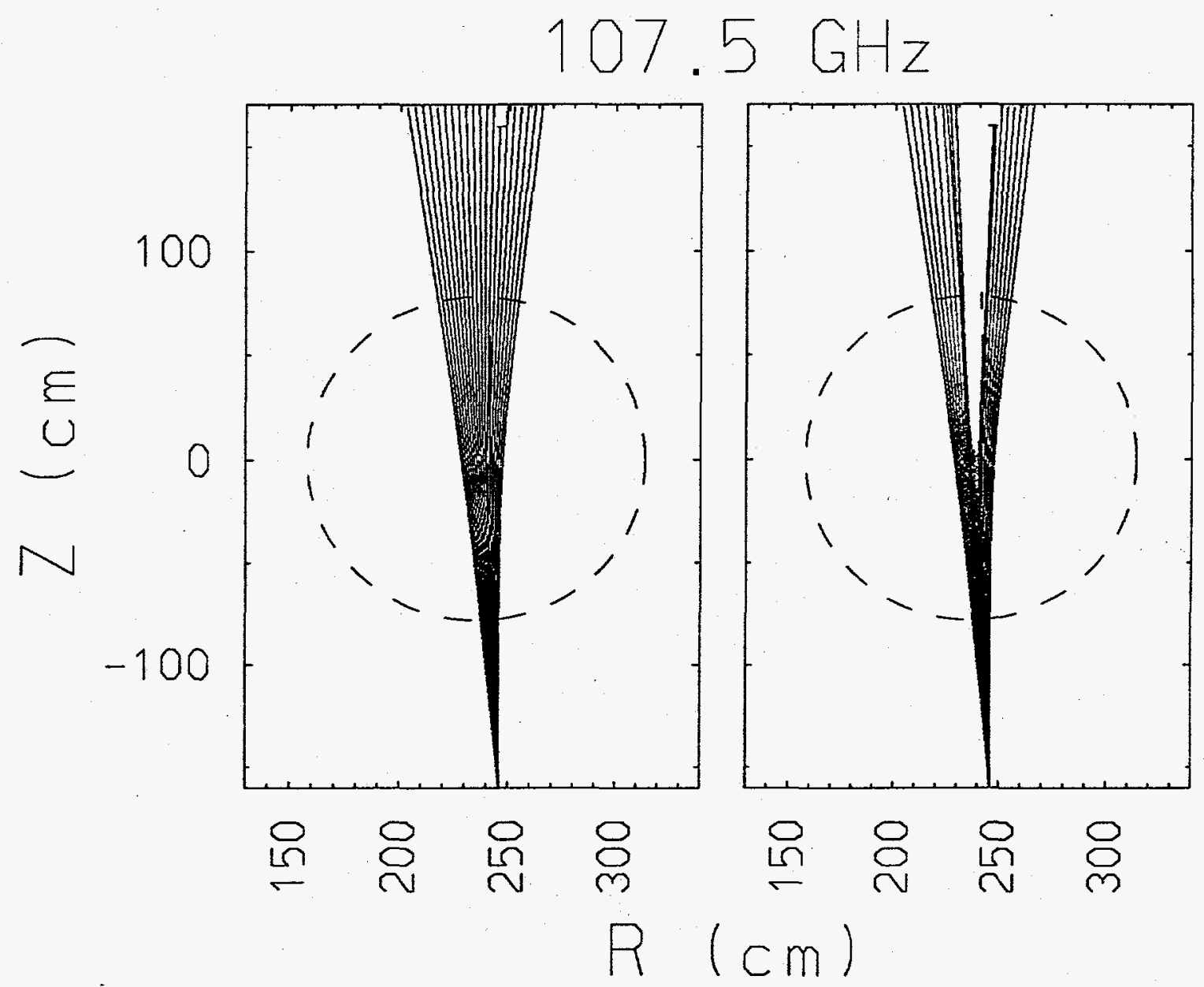

Figure 1: Ray trajectories computed within the cold-plasma (left panel) and weakly relativistic (right panel) approximations. The location of the cyclotron resonance is a barely visible, vertical dashed line. The location of the receiving horn is indicated by the short horizontal line at the top of the figures. 


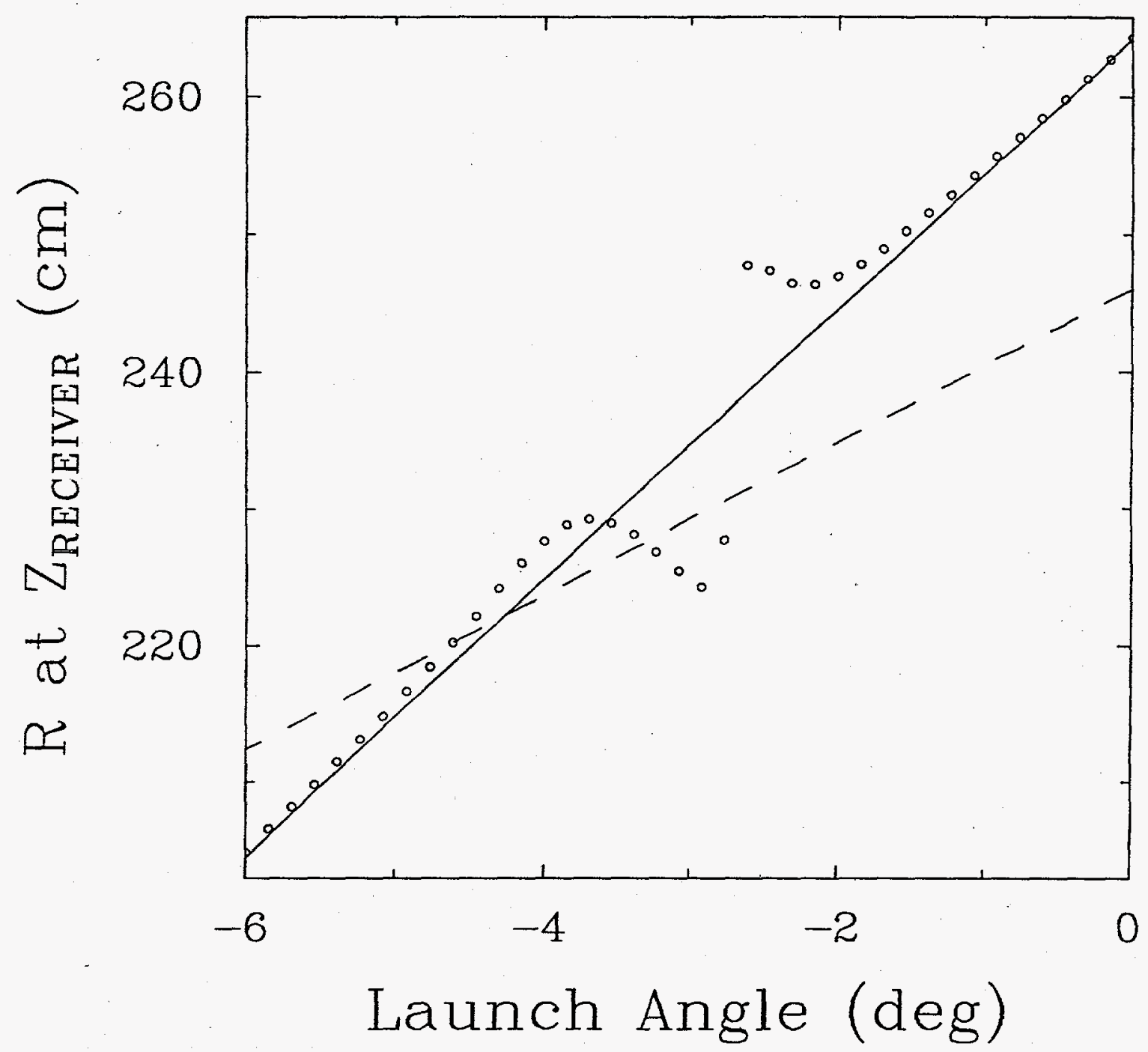

Figure 2: Radial positions $R$ of the intersection of ray trajectories with a horizontal plane $Z=160 \mathrm{~cm}$ at the height of the receiving horn, for rays launched at different angles from the vertical. The three curves are described in the text. 
the beam near that angle is strongly defocused and delivers negligible power density to a receiver at $R=247 \mathrm{~cm}$.) The two intersections determine two rays that hit the same $R$ after following different paths. These two rays may interfere either constructively or destructively, depending on the difference in the wave phase after propagation along the two paths. Such interference may explain multiple peaks, which are reminiscent of an Airy pattern, that have been observed in Tore Supra experiments. ${ }^{2,3}$ Also seen in Fig. 2 is the gap $229 \mathrm{~cm}<R<246 \mathrm{~cm}$ that is hit by negligible power density (none of our closely spaced rays). Finally, any $R$ in the interval $224 \mathrm{~cm}<R<229 \mathrm{~cm}$ is hit by power from three different launch angles. In that interval, which occurs for rays that pass on the high-field side of resonance, absorption is strong, and the focusing of rays is not expected to produce a peak in the transmitted power.

\section{Summary}

Improved methods for evaluation of Shkarofsky functions $\mathcal{F}_{q}$ that appear in the weakly relativistic dielectric tensor have been installed in the TORCH code. Rays with small $N_{n}$ can now be traced, and heating or diagnostic beams that propagate nearly perpendicular to the magnetic field can be modeled. Application to vertical thirdharmonic X-mode propagation in Tore Supra shows strong, warm-plasma refraction effects that qualitatively agree with experimental observations.

\section{Acknowledgments}

We gratefully acknowledge discussions with W.M. Nevins.

This work was performed under the auspices of the U.S. Department of Energy by LLNL under contract No. W-7405-Eng-48 and by Lehigh University under contract No. DE-FG02-92-ER5-4141. The work at MPB Technologies was supported by the Centre Canadien de Fusion Magnetique with funds from AECL, Hydro-Québec, and INRS. Partial support was also provided by the Swiss National Science Foundation. 


\section{References}

${ }^{1}$ D. Vézard, J. L. Ségui, L. Laurent, and G. Giruzzi, Phys. Plasmas 2, 876 (1995).

${ }^{2} J$. L. Ségui, An overview of ECA results in Tore Supra, these proceedings.

${ }^{3}$ J. L. Ségui, Y. Michelot, G. Giruzzi, T. Goodman, A. H. Kritz, A. Pochelon, O. Sauter, G. Smith, and M. Q. Tran, Measurement of the optical depth at the third electron cyclotron harmonic in a tokamak plasma, in Proc. 21st EPS Conf. on Controlled Fusion and Plasma Physics, edited by R. Pick, page 1004, (EPS, Geneva, 1994), Vol. II.

${ }^{4}$ A. Pochelon, K. Appert, T. P. Goodman, M. Henderson, A. Hirt, F. Hofmann, A. Kritz, J.-M. Moret, R. A. Pitts, M. Q. Tran, H. Weisen, and D. R. Whaley, Electron cyclotron resonance heating calculations for TCV, in Proc. 20th EPS Conf. on Controlled Fusion and Plasma Physics, edited by R. Pick, page 1029, (EPS, Geneva, 1993), Vol. III.

${ }^{5}$ I. P. Shkarofsky, Phys. Fluids 9, 561 (1966).

${ }^{6}$ V. Krivenski and A. Orefice, J. Plasma Phys. 30, 125 (1983).

${ }^{7}$ B. D. Fried and S. D. Conte, The Plasma Dispersion Function, (Academic Press Inc., New York, 1961).

${ }^{8} \mathrm{H}$. Bindslev, On the Theory of Thomson Scattering and Reflectometry in a Relativistic Magnetized Plasma, PhD thesis, University of Oxford, 1992, Risø National Laboratory Report Risø-R-663(EN).

${ }^{9}$ E. Borie, F. Moser, E. Räuchle, and R. A. Deike, Propagation of electron cyclotron waves in a weakly relativistic non-maxwellian plasma, in Proc. Eighth Joint Workshop on Electron Cyclotron Emission \& Electron Cyclotron Resonance Heating, edited by H. J. Hartfuss and V. Erckmann, pages 61-70, 1993, Gut Ising, Germany, Oct. 19-21, 1992. IPP Report IPP III/186, Vol. 1.

${ }^{10}$ I. P. Shkarofsky, J. Plasma Phys. 35, 319 (1986).

${ }^{11}$ G. R. Smith, Phys. Fluids 27, 1499 (1984).

${ }^{12}$ R. C. Myer, M. Porkolab, G. R. Smith, and A. H. Kritz, Nucl. Fusion 29, 2155 (1989). 